\title{
Fatigue in a cohort of geriatric patients with and without Parkinson's disease
}

\author{
F.O. Goulart ${ }^{1}$, B.A. Godke1, V. Borges ${ }^{1}$, S.M.C. Azevedo-Silva ${ }^{1}$, M.F. Mendes 3 , \\ M.S. Cendoroglo ${ }^{2}$ and H.B. Ferraz ${ }^{1}$ \\ 15etor de Transtornos do Movimento, Departamento de Neurologia, \\ 2Disciplina de Geriatria, Departamento de Medicina, Escola Paulista de Medicina, \\ Universidade Federal de São Paulo, São Paulo, SP, Brasil \\ ${ }^{3}$ Disciplina de Neurologia, Irmandade da Santa Casa de São Paulo, São Paulo, SP, Brasil \\ Correspondence to: H.B. Ferraz, Departamento de Neurologia, Rua Pedro de Toledo, 655, 04039-032 \\ São Paulo, SP, Brasil \\ E-mail: henrique_ferraz@uol.com.br
}

\begin{abstract}
We evaluated the frequency of fatigue in geriatric patients with and without Parkinson's disease (PD) and correlated it with depression and excessive daytime sleepiness. We evaluated 100 patients from Hospital São Paulo, 50 with PD from the Neurologic Outpatient Clinic and 50 with non-neurologic diseases or oncologic diseases from the Geriatric Outpatient Clinic (controls). All patients who scored 28 or more on the Fatigue Severity Scale (FSS) were considered to have fatigue. Also, all patients were submitted to a structured interview to diagnose depression by the criteria of the American Psychiatric Association (DSM-IV, 4th version) and were evaluated by the Modified Impact of Fatigue Scale and the Epworth Sleepiness Scale (ESE) to detect excessive daytime sleepiness. Demographic and disease details of all PD patients were recorded and the patients were examined and rated by the Unified Parkinson's Disease Rating Sale (UPDRS) and Hoehn-Yahr staging. Frequency of fatigue (FSS $\geq 28$ ) was $70 \%$ for PD and $22 \%$ for controls. Twenty of 35 PD patients with fatigue had concomitant depression. Compared to controls, PD patients were found more frequently to have depression by DSM-IV criteria (44 vs $8 \%$, respectively) and excessive daytime sleepiness by the ESE (44 vs 16\%), although only depression was associated with fatigue. Fatigue was more frequent among depressed PD and control patients and was not correlated with PD duration or with UPDRS motor Scores. ESE scores did not differ between patients with or without fatigue.
\end{abstract}

Key words: Parkinson's disease; Fatigue; Depression; Excessive daytime sleepiness

Received September 23, 2008. Accepted May 18, 2009

\section{Introduction}

Fatigue is a most prevalent symptom in many clinical conditions, particularly Parkinson's disease (PD). The main signs and symptoms of PD are rest tremor, muscular rigidity, akinesia and postural and balance disorders, all of them of a motor nature. Non-motor symptoms are also quite common in PD but have been neglected. Fatigue is one of the more frequent non-motor manifestations of PD and can affect 40 to $56 \%$ of the patients (1-3).

In clinical conditions where fatigue is present there is a frequent association with depression and excessive daytime sleepiness. Both depression and excessive daytime sleepiness are also frequent in PD and may coexist with fatigue but it has not been established if they have a cause and effect relationship.

A study of 313 depressed chronic patients treated and followed-up over a period of 10 years showed that fatigue was the most powerful indicator of worsening of disease symptoms (4). Even in successfully treated patients, fatigue may persist in 10 to $35 \%$ of major depression cases $(5,6)$.

Sleep disorders are also common in PD and may be present in 74 to $98 \%$ of the patients (7-9). Sleep disorders seem to be caused by neurotransmitter abnormalities other than degeneration of the dopaminergic pathway and may be associated with the use of dopaminergic agents (7). The most frequent sleep disorders in PD include insomnia, 
excessive daytime sleepiness, restless legs syndrome, REM sleep behavior disorders, and sleep apnea (10). Excessive daytime sleepiness may affect 15 to $65 \%$ of PD patients (11-14).

Since fatigue is prevalent in PD and in other clinical conditions of the elderly, one could raise the question if fatigue in PD is a specific symptom of the disease or merely a symptom associated with patient age. The aim of the present study was to detect the presence of fatigue in a cohort of geriatric patients older than 60 years with and without PD. Secondarily, we explored the relationship of fatigue with excessive daytime sleepiness and depression.

\section{Patients and Methods}

We evaluated 50 PD patients who comprised the Parkinson Group and 50 geriatric patients without PD who comprised the control group. All 100 patients were selected from the outpatient clinic of Hospital São Paulo, Universidade Federal de São Paulo (UNIFESP). PD patients were followed-up at the Movement Disorders Unit of the Department of Neurology and controls were followedup at the Geriatric outpatient clinic of the Department of Medicine.

PD patients had to be older than 60 years and were diagnosed according to the United Kingdom Parkinson's Disease Society Brain Bank Clinical Criteria (15).

Control patients were geriatric patients followed due to chronic clinical diseases such as arterial hypertension, cardiac failure, chronic respiratory diseases, digestive disorders, etc. We excluded from both groups patients younger than 60 years, those who had received any drug with antidopaminergic effects during the past 24 weeks, and also those who had any oncologic, rheumatologic or neurologic disease other than PD.

Demographic data were recorded for all patients, such as gender, age, medication in use and disease duration. All patients were submitted to a structured interview with the neurologists involved in this study, answering questions of the items of the American Psychiatric Association (DSM-IV, 4th version) criteria for depression (16). All PD patients were evaluated by the Hoehn-Yahr staging scale (17) and by the Unified Parkinson's Disease Rating Scale part 3 (UPDRS motor score) to measure motor impairment (18).

All 100 patients were evaluated by the Beck Depression Inventory (BDI) (19), Mini-Mental State Examination (20) and fatigue scales. The Epworth Sleepiness Scale was applied to all patients and those who scored more than 10 were considered to have excessive daytime sleepiness (21). We used the Fatigue Severity Scale (FSS) (22) and
Modified Fatigue Impact Scale (MFIS) to rate fatigue in our patients (23).

Both fatigue scales used had been previously validated for the Portuguese language (24). We considered fatigue to be present if patients scored 28 or more on the FSS (24).

Data were analyzed statistically by the Student $t$-test to compare two independent samples, by the chi-square or Fisher exact test, where applicable, to analyze correlation between two independent samples, and by Pearson and Spearman coefficients to quantify the linear relation and the tendency of growing between two quantitative variables, respectively.

Data were analyzed using the Statistical Package for the Social Sciences (SPSS) software, version 11.0 for Windows.

The study was approved by the Ethics Committee of Hospital São Paulo (process \#0156-06) and written informed consent was obtained from each patient.

\section{Results and Discussion}

The demographic characteristics of both groups are shown in Table 1. Women predominated in controls and the mean ages of the patients were similar in both groups. Fatigue and depression were more frequent in PD patients than in controls. PD patients had higher scores on the Epworth Sleepiness Scale.

There was no correlation between duration of PD symptoms and FSS scores. Mean UPDRS motor scores were similar for PD patients with and without fatigue.

Table 2 shows that fatigue was significantly correlated with depression as diagnosed by DSM-IV criteria in both the PD group and the control group.

The mean scores of the Epworth Sleepiness Scale for patients with and without fatigue in both groups (PD and controls) were not significantly different.

The control group had a high prevalence of women. One could argue that this could be a source of bias because depression is usually more prevalent in women. However, we observed only an $8 \%$ frequency of depression in our control patients as opposed to $44 \%$ in PD patients.

The present study showed that $70 \%$ of PD patients had fatigue, a higher frequency than usually reported in other surveys. The prevalence of fatigue among PD patients has been reported to range from 32 to $56 \%(2,3,25)$. PD patients in the early phase of symptoms may have a $30 \%$ prevalence of fatigue and this rate can rise to $50 \%$ if they are followed-up for a period of 42 weeks (26). Our PD patients had an average duration of symptoms of 6.3 years 
and this longer duration of the disease may explain the higher frequency of fatigue among them. On the other hand, there was no correlation between FSS scores and duration of symptoms. In other words, the duration of PD may be important for the development of fatigue but not for its severity.

Fatigue was observed in $22 \%$ of our control geriatric patients, which could be considered to be a high proportion of patients in comparison with the general population. However, elderly subjects have a relatively high prevalence of fatigue, especially those with clinical problems, as was the case for our control sample $(27,28)$.

Depression was detected in 44\% of PD patients and $8 \%$ of control patients, as shown in Table 1. Depression can affect 40 to $50 \%$ of PD patients and less than $10 \%$ of elderly people (29). Our PD patients with depression had higher BDI scores than the geriatric patients without PD, suggesting that depression, when present, is more severe among PD patients. However, we could also explain these findings by the small number of depressed individuals in the control group or by the choice of BDI to measure the severity of depression. BDI has many questions regarding somatic symptoms and some of them could be confounded with some non-motor symptoms of PD. PD patients have depression more frequently than patients with other chronic neurological diseases. Depression can be the first manifestation of PD and may cause functional disability by itself in patients with mild motor symptoms $(30,31)$.

Schifitto et al. (26) demonstrated that fatigue and severity of motor symptoms could be correlated. However, our study did not demonstrate a correlation between fatigue and duration or severity of PD, and the same was also reported by others $(1,2,23,32)$. Non-motor manifestations of PD, such as olfaction disorder, constipation and depression may precede motor symptoms by many years (33). Fatigue is one of these non-motor symptoms and is

Table 1. Characteristics of the Parkinson's disease and control groups.

\begin{tabular}{lccc}
\hline & Parkinson's disease & Control & Total \\
\hline Women/Men & $28 / 22^{*}$ & $43 / 7$ & $71 / 29$ \\
Age (mean in years) & 70.5 & 73.9 & 72.2 \\
Hoehn-Yahr Stage (mean) & 2.53 & - & - \\
UPDRS motor score (mean score) & 31.5 & - & - \\
Duration of Parkinson's disease (mean in years) & 6.3 & - & - \\
Mini-Mental State Examination (mean score) & 25.8 & 26.0 & 25.9 \\
Beck Depression Inventory (mean score) & $14.1^{*}$ & 5.6 & 9.8 \\
Fatigue Severity Scale (mean score) & $37.1^{*}$ & 20.4 & 28.7 \\
Total MFIS (mean score) & $36.1^{*}$ & 15.4 & 25.7 \\
Epworth Sleepiness Scale (mean score) & $9.3^{*}$ & 5.7 & 7.5 \\
Presence of fatigue according to FSS (FSS $\geq 28)$ & $35(70 \%)^{*}$ & $11(22 \%)$ & $46(46 \%)$ \\
Presence of depression according to DSM-IV & $22(44 \%)^{*}$ & $4(8 \%)$ & $26(26 \%)$ \\
With excessive daytime sleepiness (ESE $\geq 10)$ & $22(44 \%)^{*}$ & $8(16 \%)$ & $30(30 \%)$ \\
\hline
\end{tabular}

UPDRS = Unified Parkinson's Disease Rating Scale; FSS = Fatigue Severity Scale; MFIS = Modified Fatigue Impact Scale; DSM-IV = Diagnostic and Statistical Manual of Mental Disorders; ESE = Epworth Sleepiness Scale. ${ }^{*} \mathrm{P}<0.05$ compared to control group (chi-square test and Student $t$-test).

Table 2. Fatigue (FSS $\geq 28$ ) and depression (DSM-IV) in the Parkinson's disease and control groups.

\begin{tabular}{lcrrrr}
\hline & \multicolumn{2}{c}{ Without depression } & & \multicolumn{2}{c}{ With depression } \\
\cline { 2 - 3 } \cline { 5 - 6 } & Parkinson's disease & Control & & Parkinson's disease & Control \\
\hline Without fatigue & $13(26 \%)$ & $38(76 \%)$ & & $2(4 \%)$ & $1(2 \%)$ \\
With fatigue & $15(30 \%)$ & $8(16 \%)$ & & $20(40 \%)$ & $3(6 \%)$ \\
Total & $28(56 \%)$ & $46(92 \%)$ & & $22(44 \%)$ & $4(8 \%)$ \\
\hline
\end{tabular}

Data are reported as number and percent. FSS = Fatigue Severity Scale; DSM-IV = Diagnostic and Statistical Manual of Mental Disorders. Fatigue was associated with depression in both the Parkinson's disease and control groups $(P<0.05$, chi-square test). 
probably not related to dopaminergic dysfunction. This could explain why we did not correlate it with higher motor scores on the UPDRS.

Table 2 shows that depression was associated with fatigue in both the PD and control groups. A survey conducted in Norway using the same methodology as in the present study found that fatigue was present in $44 \%$ of PD patients and in $18 \%$ of control subjects (2) and that fatigue was associated with depression, as also observed here. Although fatigue and depression may be associated, treating depression does not improve fatigue (32). A Japanese study did not detect an association between fatigue and depression (34).

Excessive daytime sleepiness was found in 44\% of PD patients and in $16 \%$ of control patients. Dopaminergic agents are strongly associated with somnolence and sleep attacks and could be the cause of excessive daytime sleepiness in some of our patients (35). REM sleep behavioral disorder, restless legs syndrome and periodic movements of the legs are prevalent conditions in PD patients and are putative causes of excessive daytime sleepiness (36). In the present study, fatigue and excessive daytime sleepiness were not associated and the same was observed by others $(27,37)$.

Our study showed that fatigue was very frequent in our sample of Brazilian PD patients. Fatigue and depression are associated in both PD and geriatric patients. Although there is no known treatment for fatigue in $\mathrm{PD}$, it is important to be aware of its occurrence, so that it may be recognized and not mistaken for other motor and psychic symptoms.

\section{References}

1. Van Hilten JJ, Hoogland G, van der Velde EA, Middelkoop HA, Kerkhof GA, Roos RA. Diurnal effects of motor activity and fatigue in Parkinson's disease. J Neurol Neurosurg Psychiatry 1993; 56: 874-877.

2. Karlsen K, Larsen JP, Tandberg E, Jorgensen K. Fatigue in patients with Parkinson's disease. Mov Disord 1999; 14: 237-241.

3. Herlofson K, Larsen JP. The influence of fatigue on healthrelated quality of life in patients with Parkinson's disease. Acta Neurol Scand 2003; 107: 1-6.

4. Wick JY, LaFleur J. Fatigue: implications for the elderly. Consult Pharm 2007; 22: 566-576.

5. Fava GA, Grandi S, Zielezny M, Canestrari R, Morphy MA. Cognitive behavioral treatment of residual symptoms in primary major depressive disorder. Am J Psychiatry 1994; 151: 1295-1299.

6. Nierenberg AA, Keefe BR, Leslie VC, Alpert JE, Pava JA, Worthington JJ III, et al. Residual symptoms in depressed patients who respond acutely to fluoxetine. J Clin Psychiatry 1999; 60: 221-225.

7. Nausieda PA, Weiner WJ, Kaplan LR, Weber S, Klawans $\mathrm{HL}$. Sleep disruption in the course of chronic levodopa therapy: an early feature of the levodopa psychosis. Clin Neuropharmacol 1982; 5: 183-194.

8. Lees AJ, Blackburn NA, Campbell VL. The nighttime problems of Parkinson's disease. Clin Neuropharmacol 1988; 11: $512-519$

9. Kumar S, Bhatia M, Behari M. Excessive daytime sleepiness in Parkinson's disease as assessed by Epworth Sleepiness Scale (ESS). Sleep Med 2003; 4: 339-342.

10. Stacy M. Sleep disorders in Parkinson's disease: epidemiology and management. Drugs Aging 2002; 19: 733-739.

11. Factor SA, McAlarney T, Sanchez-Ramos JR, Weiner WJ. Sleep disorders and sleep effect in Parkinson's disease. Mov Disord 1990; 5: 280-285.

12. Van Hilten JJ, Weggeman M, van der Velde EA, Kerkhof GA, van Dijk JG, Roos RA. Sleep, excessive daytime sleepi- ness and fatigue in Parkinson's disease. J Neural Transm Park Dis Dement Sect 1993; 5: 235-244.

13. Tandberg E, Larsen JP, Karlsen K. Excessive daytime sleepiness and sleep benefit in Parkinson's disease: a community-based study. Mov Disord 1999; 14: 922-927.

14. Arnulf $\mathrm{I}$. Excessive daytime sleepiness in parkinsonism. Sleep Med Rev 2005; 9: 185-200.

15. Hughes AJ, Daniel SE, Kilford L, Lees AJ. Accuracy of clinical diagnosis of idiopathic Parkinson's disease: a clinicopathological study of 100 cases. J Neurol Neurosurg Psychiatry 1992; 55: 181-184.

16. American Psychiatric Association. Diagnostic and statistical manual of mental disorders (DSM-IV). 4th edn. Washington: APA; 1994

17. Hoehn MM, Yahr MD. Parkinsonism: onset, progression and mortality. Neurology 1967; 17: 427-442.

18. Fahn S, Elton RL, Members of the UPDRS Development Committee. Unified Parkinson's Disease Rating Scale. In: Fahn S, Marden CD, Calne D, Goldstein M (Editors), Recent developments in Parkinson's disease. Vol. 2. Florham Park: Macmillan Healthcare Information; 1987. p 153-163.

19. Beck AT, Ward CH, Mendelson M, Mock J, Erbaugh J. An inventory for measuring depression. Arch Gen Psychiatry 1961; 4: 561-571.

20. Folstein MF, Folstein SE, McHugh PR. "Mini-mental state". A practical method for grading the cognitive state of patients for the clinician. J Psychiatr Res 1975; 12: 189-198.

21. Johns MW. A new method for measuring daytime sleepiness: the Epworth sleepiness scale. Sleep 1991; 14: 540545.

22. Krupp LB, LaRocca NG, Muir-Nash J, Steinberg AD. The fatigue severity scale. Application to patients with multiple sclerosis and systemic lupus erythematosus. Arch Neurol 1989; 46: 1121-1123.

23. Multiple Sclerosis Council for Clinical Practice Guidelines. Fatigue and multiple sclerosis: evidence-based management strategies for fatigue in multiple sclerosis. Washing- 
ton: Paralyzed Veterans of America; 1998.

24. Mendes MF, Tilbery CP, Felipe E. [Fatigue and multiple sclerosis: preliminary study of 15 patients with self-reported scales]. Arq Neuropsiquiatr 2000; 58: 467-470.

25. Friedman J, Friedman H. Fatigue in Parkinson's disease. Neurology 1993; 43: 2016-2018.

26. Schifitto G, Friedman JH, Oakes D, Shulman L, Comella $\mathrm{CL}$, Marek K, et al. Fatigue in levodopa-naive subjects with Parkinson disease. Neurology 2008; 71: 481-485.

27. Havlikova E, van Dijk JP, Rosenberger J, Nagyova I, Middel $\mathrm{B}$, Dubayova T, et al. Fatigue in Parkinson's disease is not related to excessive sleepiness or quality of sleep. $J$ Neurol Sci 2008; 270: 107-113.

28. Poluri A, Mores J, Cook DB, Findley TW, Cristian A. Fatigue in the elderly population. Phys Med Rehabil Clin N Am 2005; 16: $91-108$.

29. Tandberg E, Larsen JP, Aarsland D, Cummings JL. The occurrence of depression in Parkinson's disease. A community-based study. Arch Neurol 1996; 53: 175-179.

30. Nuti A, Ceravolo R, Piccinni A, Dell'Agnello G, Bellini G, Gambaccini G, et al. Psychiatric comorbidity in a population of Parkinson's disease patients. Eur J Neurol 2004; 11: 315-
320.

31. Ravina B, Camicioli R, Como PG, Marsh L, Jankovic J, Weintraub $D$, et al. The impact of depressive symptoms in early Parkinson disease. Neurology 2007; 69: 342-347.

32. Friedman $\mathrm{JH}$, Friedman $\mathrm{H}$. Fatigue in Parkinson's disease: a nine-year follow-up. Mov Disord 2001; 16: 1120-1122.

33. Braak H, Ghebremedhin E, Rub U, Bratzke H, Del TK. Stages in the development of Parkinson's disease-related pathology. Cell Tissue Res 2004; 318: 121-134.

34. Abe K, Takanashi M, Yanagihara T. Fatigue in patients with Parkinson's disease. Behav Neurol 2000; 12: 103-106.

35. Biglan KM, Holloway RG Jr, McDermott MP, Richard IH. Risk factors for somnolence, edema, and hallucinations in early Parkinson disease. Neurology 2007; 69: 187-195.

36. Larsen JP, Tandberg E. Sleep disorders in patients with Parkinson's disease: epidemiology and management. CNS Drugs 2001; 15: 267-275.

37. Havlikova E, Rosenberger J, Nagyova I, Middel B, Dubayova T, Gdovinova Z, et al. Impact of fatigue on quality of life in patients with Parkinson's disease. Eur J Neurol 2008; 15: 475-480. 